\title{
EMPIRICAL COMPARISON OF HUMAN BEHAVIOR AND PERFORMANCE WITH DIFFERENT DISPLAY DEVICES FOR VIRTUAL ENVIRONMENTS
}

\author{
Doug A. Bowman ${ }^{1}$, Ameya Datey ${ }^{1}$, Young Sam Ryu ${ }^{2}$, Umer Farooq ${ }^{1}$, and Omar Vasnaik ${ }^{1}$ \\ ${ }^{1}$ Department of Computer Science \\ ${ }^{2}$ Grado Department of Industrial and Systems Engineering \\ Virginia Polytechnic Institute and State University \\ Blacksburg, Virginia
}

\begin{abstract}
Although a wide range of display devices is used in virtual environment (VE) systems, no guidelines exist to choose an appropriate display for a particular VE application. Our goal in this research is to develop such guidelines on the basis of empirical results. In this paper, we present a preliminary experiment comparing human behavior and performance between a head-mounted display (HMD) and a four-sided spatially immersive display (SID). In particular, we studied users' preferences for real vs. virtual turns in the VE. The results indicate that subjects have a significant preference for real turns in the HMD and for virtual turns in the SID. The experiment also found that females are more likely to choose real turns than males. We suggest that HMDs are an appropriate choice when users perform frequent turns and require spatial orientation.
\end{abstract}

\section{INTRODUCTION}

The visual display system is an integral part of a virtual environment (VE) system. In fact, many VEs are characterized by the display device they use (e.g. a "CAVE application" or a "head-mounted display system"). Although we have some intuitive understanding of the relative strengths and weaknesses of some of the most common VE display devices, there are few empirical results to back up these intuitions. As Fred Brooks noted in his review of the current state of the art in VEs, one of the most crucial challenges in the field is "choosing which display best fits each application" (Brooks, 1999). Our ultimate goal is to develop guidelines that create a mapping between an application and a display, or, more specifically, between an application's requirements and a display, for the purpose of improving user task performance and usability. For example, a useful result might be, "If users' task performance in an application requires the highest level of perceived immersion, use a spatially immersive display."

We present a preliminary study aimed at producing this type of guideline. We compare users' behavior and performance with a head-mounted display (HMD) to those with a spatially immersive display (SID) to study the effects of manual rotation vs. natural rotation of the user's viewpoint. This experiment provides some insight into the subtle differences of human performance between the display types, and suggests many interesting lines of future work.

\section{RELATED WORK}

There has been a great deal of effort in the VE community aimed at developing new displays (e.g. Meyer and Barr, 1999) and improving existing display types (e.g. Kijima and Ojika, 1997). However, there is little work that objectively compares human behavior and performance in different VE displays. A SIGGRAPH '96 panel (Lantz, 1996) asked prominent VE researchers to make a case for HMDs or SIDs as the "future of virtual reality." Panelists in general proposed that displays should be chosen based on the tasks and requirements of a particular application, but no empirical results were given.

Our experiment focuses on natural (physical) viewpoint rotation vs. manual rotation, similar to Pausch et al (Pausch, Shackelford, and Proffitt, 1993). Research suggests that physical motion allows better estimates of the magnitude of rotation (Bakker, Werkhoven, and Passenier, 1998). Chance et al. (1998) found that natural turns produced significantly higher levels of spatial orientation than manual turns, a fact which has important implications for the choice of $\mathrm{VE}$ displays given the results of our experiment. The present study differs from the earlier work because it investigates both required and elective manual turns, and because it compares two completely different display types.

\section{DISPLAY DEVICES}

Three-dimensional (3D) visual display technology for use in virtual environments has become quite varied. For many years, the head-mounted display (HMD) was considered the most common VE display. A range of new display devices has appeared recently that promises to be useful for VE systems. These include workbench displays, desktop stereoscopic displays, retinal displays, and spatially immersive displays (SIDs). In this section we explore the characteristics of the two display types compared in our experiment.

\section{Head-mounted displays}

The HMD (figure 1) is considered the canonical VE display. This device usually consists of two LCD or CRT screens that are mounted in a helmet-like device so that they are fixed relative to the wearer's eye position. This device portrays the virtual world by obtaining the user's head position and orientation from a tracking system. Imagery is magnified with a set of optics.

There are several attributes of HMDs that may have an effect on a user's performance (Davis, 1996). First, HMDs 
may present biocular (same image to both eyes) or stereoscopic (different images to each eye) imagery. Second, HMDs come in a wide range of resolutions. Resolution usually trades off with field of view (FOV), which is measured in degrees of horizontal visual angle. A lower FOV results in "tunnel vision" and may decrease immersion, but higher FOVs involve spreading out the available pixels, which can decrease resolution and introduce distortion. Finally, there are ergonomic issues related to HMDs such as the display's size and weight and the ability to adjust various visual parameters.

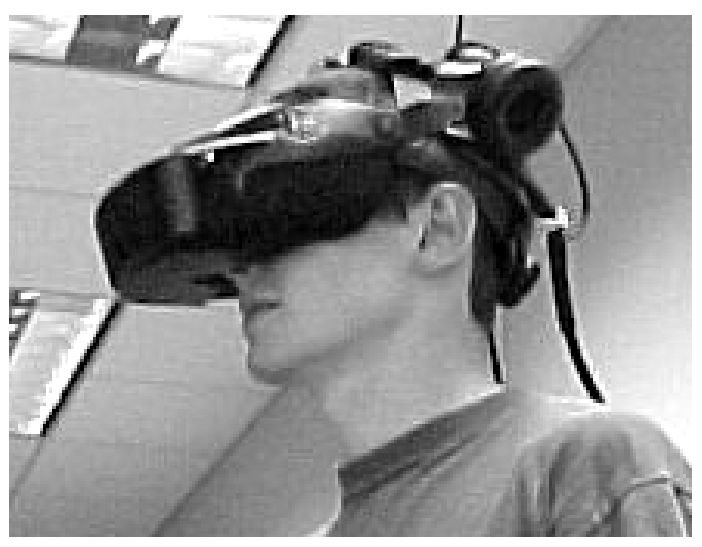

Figure 1. User wearing a head-mounted display (HMD)

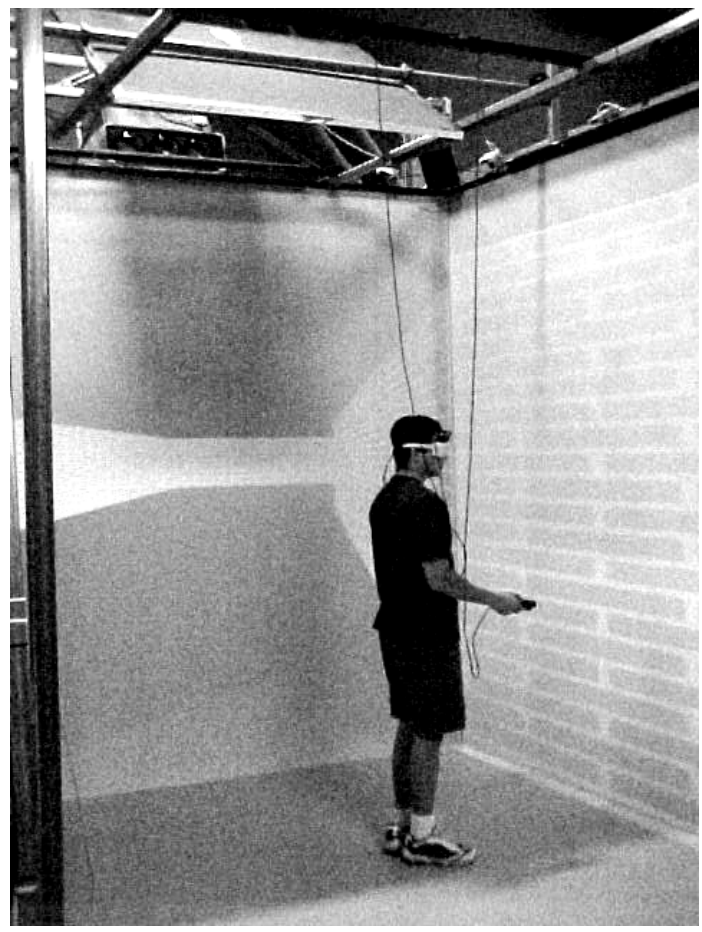

Figure 2. User within a spatially immersive display (SID)

\section{Spatially immersive displays}

Rather than using a single set of displays that follow the user's head movements, spatially immersive displays (SIDs) use multiple displays that surround most of the user's field of view (figure 2). The most common example of a SID is the CAVE (Cruz-Neira, Sandin, and DeFanti, 1993). CAVEs generally use four large back-projected screens set at right angles (three walls and a floor), onto which stereo graphics are projected. The user wears stereo glasses and a head tracker. The tracker allows the 3D environment to be rendered from the user's point of view and allows the user to move naturally to change his viewpoint.

A high level of immersion is the most attractive feature of CAVEs and other SIDs. The user is also less encumbered than with HMD systems. In addition, the FOV in a CAVE (assuming the user looks at the front wall) can be more than 180 degrees, which matches the eye's perceptual limit (this FOV is usually not achieved, however, due to the limitation of the FOV through the stereo glasses).

On the other hand, CAVEs may be less immersive than HMDs because of the two missing surfaces of the cube (back wall and ceiling) that break the illusion of being within a $3 \mathrm{D}$ space. In a standard CAVE, the user must have some method for rotating the environment in order to see objects behind or above them. Also, the projected graphics tend to be less bright, and thus require a darkened room. Finally, CAVEs are currently many orders of magnitude more expensive than HMD-based systems, making them impractical for many potential users.

\section{NATURAL VS. MANUAL ROTATION}

As noted above, most SIDs produce excellent perceived immersion because of their high resolution, excellent stereo, and wide field of view. However, the most common SIDs do not provide complete physical immersion, since two sides of the six-sided cube are usually missing. In such displays, users cannot physically turn 360 degrees to view the world all around them. Rather, they must manually rotate the world to see what is behind or above them. In HMDs, on the other hand, perceived immersion may be less, due to a low field of view, lower resolution, and so on, but physical immersion is complete - the user sees the virtual world no matter what direction she looks.

To test the implications of this tradeoff in the two displays, we implemented a series of corridors in which users could choose between natural and manual turns. Since manual turns are less natural and reduce spatial orientation, and since they might be less efficient, we expected that SID users would only use manual rotation when it was required to avoid facing the missing wall. We further conjectured that HMD users would not use the manual rotation option frequently. By collecting empirical data about the way users turn, we could determine appropriate interaction techniques for navigation in various VE applications based on the type of display.

\section{METHOD}

\section{Subjects}

The experiment was conducted with 18 subjects. Out of these, experiments with two subjects were aborted due to 
discomfort on the part of the subject. Consequently, 16 subjects (eight male, eight female) completed the experiment.

\section{Apparatus}

The HMD condition used a Virtual Research V8 HMD with biocular (non-stereo) graphics, VGA resolution, and a 60degree diagonal field of view. The SID condition in this experiment used a Fakespace CAVE. It is a theater of 10x10x9 feet, made up of three rear-projection screens for the front, right and left walls and a down-projection screen for the floor. Head tracking in both conditions was provided by an Intersense IS-900 tracking system. A joystick mounted on the Intersense 'wand' tracker in both conditions allowed the user to move forward/backward and to rotate the viewpoint manually.

\section{Environment}

Eight corridors were created and used in both the HMD and CAVE conditions. The walls had a texture of a brick and were made higher than the user, so that the user could not see over the walls and get any additional spatial information. Corridors had no choice points, so there was no decision making about the path to be followed. All turns were at right angles. Figure 3 shows a typical view within a virtual corridor.

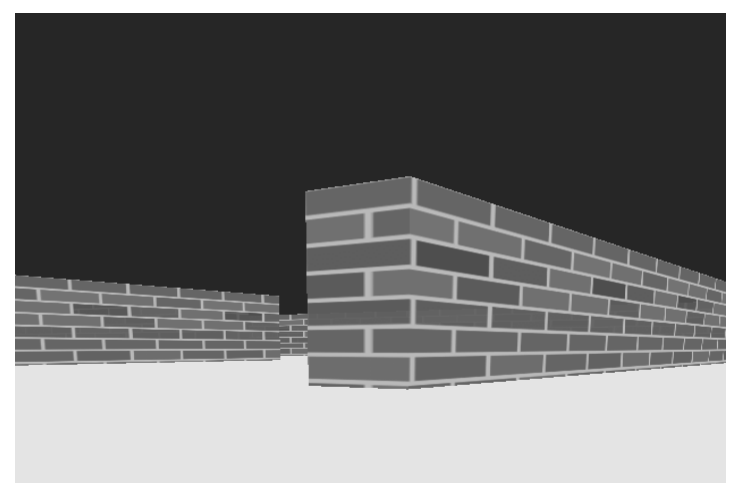

Figure 3. User's view of a virtual corridor

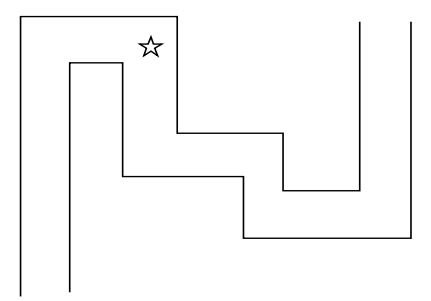

Figure 4. Top-down view of a virtual corridor with mandatory manual rotation

The subject could press the joystick forward or backward to move forward or backward respectively in the direction he was facing (gaze-directed steering). The subject could turn his head or entire body naturally while making a turn. We call this natural rotation. The subject could also choose to use the joystick to rotate the world about its vertical axis; this is manual rotation. Finally, the subject could combine both the techniques while making a turn. This is combination rotation.

Figure 4 shows a map-like view of a virtual corridor. In a 4-wall CAVE, the user would be forced to rotate the environment manually at the position marked by a star, assuming the user starts by facing the front wall of the CAVE. This forced manual turn is referred to as mandatory manual rotation.

\section{Experimental design}

The experiment used a within-subjects design. The single independent factor in this experiment was the display device (HMD and CAVE). Each subject participated in two sessions, one for each display device. The sessions were conducted on the same day, one immediately after the other. The order of sessions was alternated; half of the subjects started with the HMD, and the rest started with the CAVE. The sequence of corridors was counterbalanced by the use of a Latin Square design.

The number of turns using each of the techniques was observed and recorded. Also, the time needed to complete each trial was recorded. These two factors were the dependent variables.

\section{Procedure}

The subjects were first given a demographic questionnaire. This questionnaire asked for information such as age, gender, and occupation (or major field of study), and for information on the subject's use of computers and prior experience with VEs.

Subjects were then placed in a practice corridor to get them acquainted with the setup. In the actual trials, the subjects were told to try and navigate to the end of the corridor as quickly and efficiently as they could. Subjects completed eight trials using each device, and were free to use natural, manual, or combination rotation at each turn. Evaluators closely watched both the subject and the graphics display to determine the technique used for each turn.

\section{RESULTS}

\section{Rotation types}

Figure 5 shows the percentage of the different types of rotations with the two display devices. It is quite clear that the percentage of natural rotations is smaller in the CAVE than the $\mathrm{HMD}$, whereas the percentage of manual rotations is greater in the CAVE than in the HMD. Based on a single-factor ANOVA, we found that display type significantly affected the percentage of natural turns and the percentage of combination turns $(\mathrm{F}(1,30)=7.40, \mathrm{p}<0.02$ and $\mathrm{F}(1,30)=10.09, \mathrm{p}<0.005$ respectively). Combining these results with the results from Chance et al. (1998), we can conclude that HMD users are 
more likely to maintain spatial orientation than CAVE users in an environment where turning is frequent.

There was a noticeable difference in turning preference based on gender. Females seemed to prefer natural rotation, and tried to use it as much as possible. Considering only the female subjects, only the natural turns were significantly affected by display type $(\mathrm{F}(1,14)=7.04, \mathrm{p}<0.02)$, whereas in the case of male subjects, significant results were observed on combination turns $(\mathrm{F}(1,14)=8.30, \mathrm{p}<0.02)$.
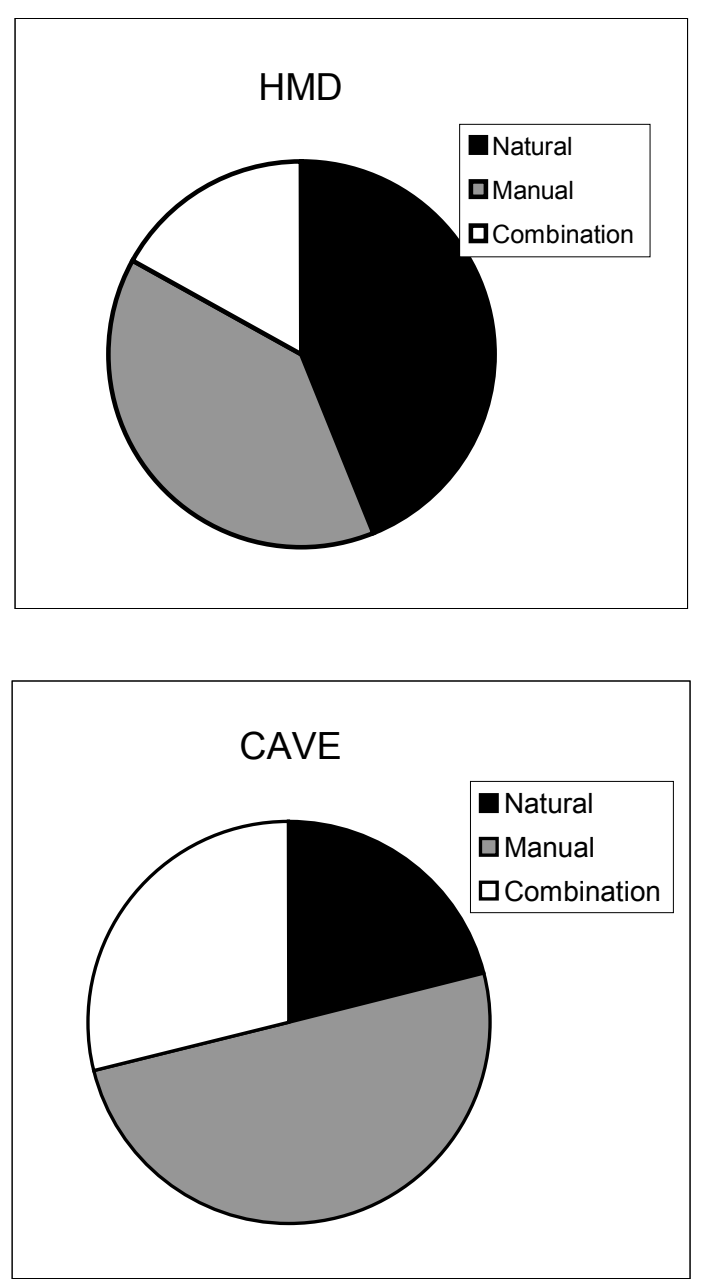

Figure 5. Percentage of different types of rotations in HMD and CAVE

The results show that natural rotation is the preferred technique in the HMD. In the CAVE condition, subjects used manual turns at places where manual turning was not mandatory. The CAVE's missing back wall often came as a surprise to subjects who turned naturally in the CAVE, realized the wall was missing, and then used manual rotation. After such an episode some subjects then continued using manual rotation even when it was not required.

Figure 6 compares the two display devices in terms of elective (non-mandatory) manual rotations. These ratios were calculated by dividing the total number of non-mandatory manual rotations by the total number of possible nonmandatory manual rotations for each corridor (in the HMD, all manual rotations are non-mandatory, and every turn is a possible non-mandatory manual turn; in the CAVE, only those turns where the user had a choice between natural, manual, and combination were considered). The figure shows that in a majority of the corridors, subjects chose to perform manual rotation more often in the CAVE even when it was not required. Overall, however, the difference was small: HMD users elected to use manual rotation 39 percent of the time, while CAVE users elected to do so 41 percent of the time.

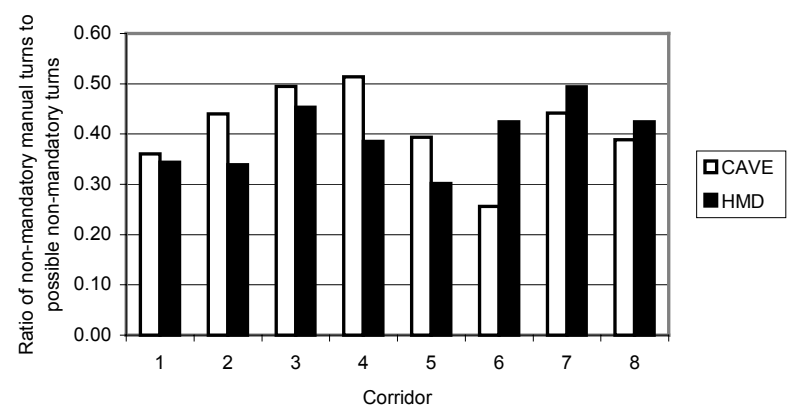

Figure 6. Percentage of elective manual rotations in the two display devices for each of the corridors

Although not significant, we also saw a trend towards an ordering effect of the two displays. Subjects who used the HMD first also used more natural turning in the CAVE. Subjects who started with the CAVE preferred more manual rotation even in the HMD.

\section{Time for traversal}

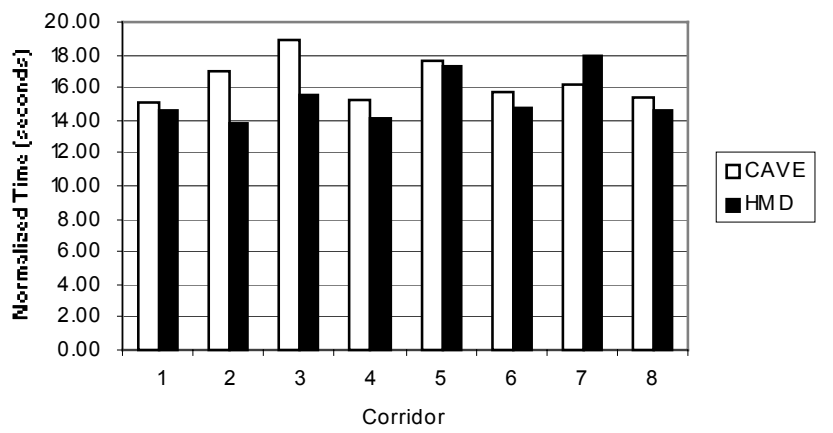

Figure 7. Average time (normalized) for traversal through each of the corridors

Figure 7 shows the evaluation of the time for traversal through the corridors. Time has been normalized to control for the effect of corridor length. It can be seen that the average time in the HMD was less than the average time in the CAVE in most of the corridors. Overall, it took an average of 15.32 seconds to traverse a corridor in the HMD and an average of 16.37 seconds in the CAVE. Although this was a strong trend, there was not a significant difference $(\mathrm{F}(1,30)=3.46, \mathrm{p}<$ $0.08)$. In the case of natural turns, the proprioceptive sense of the subject allows fast natural turns without the loss of spatial orientation. Even though manual turns could be implemented 
such that they were faster than natural turns, this increased speed of turning would likely disorient the user.

\section{CONCLUSIONS AND FUTURE WORK}

We have presented the results of an empirical evaluation comparing human performance using different virtual environment displays. The experiment showed that HMD users are significantly more likely than CAVE users to use natural rotation in a VE. This will produce higher levels of spatial orientation, and may make navigation more efficient. We also found a gender difference in rotation technique preference. Based on this experiment, we can posit an initial guideline: For VE applications involving navigation through enclosed spaces and frequent turning, choose an HMD with head tracking to provide increased efficiency and spatial orientation to users.

In the future, we plan further comparative explorations of VE display types, with the goal of producing a set of guidelines facilitating the mapping between applications and displays. The current study in particular suggests three followon experiments. First, the missing sides in SIDs may also have an effect on the sense of presence. We can test this using presence questionnaires (Witmer and Singer, 1998) or more objective measures, such as memory for object locations. Second, some authors have suggested that non-isomorphic rotations could be used in the CAVE to allow a 360-degree view even with a missing back wall (LaViola et al, 2001). It would be instructive to test the effects of such a technique on spatial orientation. There is much work to be done in this area, but the results of such experiments should allow VE developers to choose effective visual display devices for their applications.

\section{REFERENCES}

Bakker, N., Werkhoven, P., and Passenier, P. (1998). Aiding Orientation Performance in Virtual Environments with Proprioceptive Feedback. In Proceedings of IEEE Virtual Reality Annual International Symposium, Atlanta, Georgia: IEEE Computer Society Press, 28-35.

Brooks, F. (1999). What's Real About Virtual Reality?, IEEE Computer Graphics \& Applications, 19(6), 16-27.

Chance, S., Gaunet, F., Beall A. C., and Loomis J. M. (1998). Locomotion Mode Affects the Updating of Objects Encountered During Travel, Presence: Teleoperators and Virtual Environments, 7(2), 168-178.

Cruz-Neira, C., Sandin, D., and DeFanti, T. (1993). SurroundScreen Projection-Based Virtual Reality: The Design and Implementation of the CAVE. In Proceedings of ACM SIGGRAPH 1993.

Davis, E. (1996). Visual Requirements in HMDs: What Can We See and What Do We Need to See?, In Melzer, J. and Moffitt, K. (Ed.) Head-Mounted Displays: Designing for the User, McGraw-Hill: New York, 207249.

Kijima, R. and Ojika, T. (1997). Transition Between Virtual Environment and Workstation Environment with Projective Head Mounted Display. In Proceedings of
IEEE Virtual Reality Annual International Symposium, Albuquerque, New Mexico: IEEE Computer Society Press, 130-137.

Kruger, W., Bohn, C. A., Frohlich, B., Schuth, H., Strauss, W., and Wesche, G. (1995). The Responsive Workbench: A Virtual Work Environment. IEEE Computer, 28(7), 4248.

Lantz, E. (1996). The Future of Virtual Reality: Head Mounted Displays versus Spatially Immersive Displays. In Proceedings of SIGGRAPH: ACM Press, 485-486.

LaViola, J., Feliz, D. A., Keefe, D. F., Zeleznik R. C. (2001). Hands-Free Multi-Scale Navigation in Virtual Environments. In Proceedings of ACM Symposium on Interactive $3 D$ Graphics, Research Triangle Park, North Carolina, 9-15.

Meyer, M. and Barr, A. (1999). ALCOVE: Design and Implementation of an Object-Centric Virtual Environment. In Proceedings of IEEE Virtual Reality, Houston, Texas: IEEE Computer Society Press, 46-52.

Pausch, R., Shackelford, M., and Proffitt, D. (1993). A User Study Comparing Head-Mounted and Stationary Displays. In Proceedings of the IEEE Symposium on Research Frontiers in Virtual Reality, 41-45.

Witmer, B. and Singer, M. (1998). Measuring Presence in Virtual Environments: A Presence Questionnaire, Presence: Teleoperators and Virtual Environments, 7(3), 225-240. 